\title{
Nuevos indicadores de riesgo cardiovascular
}

\section{Dr. Antonio Arteaga Llona}

Departamento de Nutrición, Diabetes \& Metabolismo MED.PUC.

Recibido el 14 de Octubre de 2009, aceptado el 6 de noviembre de 2009

Rev Chil Cardiol 2009; 28: 381-384

Acevedo y cols ${ }^{1}$ presentan en este número de la Revista una investigación a nivel nacional en población adulta, en que se destaca que el grosor de la íntimamedia carotídea (CIMT) se incrementa en relación a la carga de factores de riesgo clásicos y en aquellos que presentan Síndrome Metabólico (SMet), y Proteina C reactiva ultrasensible (ePCR), lo que apoyaría el rol predictor cardiovascular de estos últimos indicadores.

En el análisis de la razón de chance (OR) de presentar un CIMT carotídeo mayor al percentil 75 , no ajustado y ajustado, por las variables estudiadas, se destaca el rol del SM asociado, o no, a ePCR elevada, por sobre el puntaje de Framingham.

Si bien el SM ha sido catalogado como uno de los problemas más fascinantes e inquietantes de la medicina actual2, aún persisten controversias y problemas no resueltos. En el momento actual se le define como el enclaustramiento de una constelación de marcadores, y se le describe como de alta prevalencia, y con creciente relevancia en varias áreas de la salud, que rebasan el área cardiovascular y de la diabetes mellitus (DM).

Considerando, específicamente, su relación con el riesgo cardiovascular, se ha discutido la utilidad clínica de identificar a un síndrome, en el contexto de poseer un mayor valor predictivo cardiovascular, que la sumatoria de sus componentes aislados 3 .

Acevedo y cols, en su modelo, nos sugiere que efectivamente el reconocer el SMet, mejora el OR de presentar valores de CIMT por sobre el P75 en relación al puntaje de Framingham, el cual considera para su estimación la sumatoria de los factores de riesgo cardiovascular.

Existe una diversidad de recomendaciones para la identificación del SM en clínica, lo cual deriva en gran parte del desconocimiento de su etiopatogenia. En la actualidad se destaca en su génesis a la interacción de resistencia insulínica y obesidad abdominal.

No es posible descartar el rol de factores genéticos en la etiopatogenia del SM, efectivamente, se reconoce que la Diabetes Mellitus Tipo 2 (DM2), patología de elevada prevalencia en el humano y que tiene un fuerte componente genético, presenta a la resistencia insulínica y a un defecto de secreción insulínica como los principales eventos etiopatogénicos.

Actualmente, la obesidad abdominal aparece como el factor más destacado, en vista de los resultados observados en cirugía bariátrica, en que en estrecha asociación con la magnitud de la reducción de peso, se observa una normalización de los todos los parámetros incluidos para identificar el SM.

La obesidad abdominal es reconocida actualmente como una condición pro-inflamatoria, que lleva a una compleja interacción entre los mediadores inflamatorios, resistencia insulínica y anomalías metabólicas.

Afortunadamente, a pesar de los distintos criterios para el diagnóstico, la capacidad predictiva de DM y patología cardiovascular no difiere en forma significativa 4 . Existe un principio de acuerdo entre los grupos de expertos 5 que componen el National Cholesterol Education Program (NCEP), Internacional Diabetes Federation (IDF), la American Diabetes Association (ADA) y American Association of Clinical

Correspondencia: Dr. Antonio Arteaga Llona

Departamento de Nutrición, Diabetes y Metabolismo

Pontificia Universidad Católica de Chile, Santiago

Correo Eléctronico:aarteaga@med.puc.cl 
Endocrinology (AACE), en identificar al síndrome metabólico, cuando coexisten 3 o más factores de los siguentes: perímetro de circunferencia abdominal (pCC) elevado, C-HDL $<40 \mathrm{mg} / \mathrm{dl}$ en hombre y $<50 \mathrm{mg}$. en mujer, Triglicéridos $\geq$ a $150 \mathrm{mg} / \mathrm{dl}$, Presión arterial $\geq 130 / 85 \mathrm{mmHg}$ y glicemia de ayuno $\geq 100 \mathrm{mg} / \mathrm{dl}$.

Una recomendación adicional de este grupo de expertos fue la necesidad de definir el punto de corte del pCC para identificar obesidad abdominal a nivel local. Lo que fue estudiado por nuestro grupo 6 en base a una población adulta representativa de Chile (Encuesta Nacional de Salud MINSAL 2003), definiendo el valor de $88 \mathrm{~cm}$. para el hombre y $83 \mathrm{~cm}$. para la mujer

En estudios prospectivos, utilizando variados criterios para identificar el SM, éste incrementó el Riesgo Relativo (RR) de presentar eventos cardiovasculares a 1.74 y de DM2 a 2.997. Destaca un estudio prospectivo en población China ${ }^{8}$, en que la identificación del SM en pacientes con disglicemia y diabetes clínica, se siguió de una significativa mayor incidencia de eventos cardiovasculares en relación a los que no presentaban SM.

Por lo señalado me parece que los médicos generales, internistas y especialistas relacionados con el área cardiovascular, deberían identificar el $\mathrm{SM}$, utilizando el criterio consensuado explicitado. Por un lado, para establecer el nivel de riesgo cardiovascular del paciente, como lo sugieren miembros del comité del NCEP ATP III ${ }^{9}$, que señalan como $2^{\circ}$ objetivo, después del C-LDL, el enfrentar el SM, y definen con riesgo máximo a un paciente coronario con SM. Por otro lado, para enfatizar la necesidad de obtener cambios persistentes en el estilo de vida del paciente, ya que la reducción y mantención de un peso saludable y la realización de una actividad física programada, constituyen las medidas más efectivas para enfrentar el SM.

Si bien la utilización de fármacos insulinosensibilizadores como la metformina y glitazonas, mejoran la sensibilidad a la insulina y previenen en forma significativa la incidencia de diabetes mellitus clínica en adultos con intolerancia a la glucosa10, su efectividad nos es mayor que los cambios de estilo de vida y no existen estudios de prevención cardiovascular prospectivos al respecto. Sólo en estudios observacionales, la metformina se ha asociado en forma consistente a un menor riesgo cardiovascular, lo que la ha llevado a ser considerada como la droga de elección junto a los cambios del estilo de vida en el tratamiento inicial de la DM2.

La identificación de los componentes del SM está al alcance del médico en general, ya que utiliza indicadores habituales en la practica médica (Ver recuadro).

Criterio actual consensuado para identificar al síndrome metabólico en Clínica (2008) Diagnóstico con 3 o más atributos:

$\begin{array}{ll}\text { Perímetro abdominal }(\mathrm{pCC})^{*} & \text { Para hombres } \geq 88 \mathrm{~cm} \\ & \text { Para mujeres } \geq 83 \mathrm{~cm} \\ \text { Triglicéridos séricos } & \geq 150 \mathrm{mg} / \mathrm{dl} \\ \text { Colesterol de HDL } & \text { Para hombres }<40 \mathrm{mg} / \mathrm{dl} \\ & \text { Para mujeres }<50 \mathrm{mg} / \mathrm{dl} \\ \text { Presión arterial } & \geq 130 / 85 \mathrm{mmHg} \\ & 0 \text { en tratamiento } \\ \text { Glicemia en ayunas } & \geq 100 \mathrm{mg} / \mathrm{dl}\end{array}$

*Valores estimados para población adulta Chilena 
Sólo requiere incorporar al equipo de diagnóstico del médico, una cinta métrica flexible para medir el pCC, utilizando como punto de referencia la línea axilar media entre el reborde costal y la espina ilíaca anterosuperior. Si bien existen controversias acerca del nivel de medición, el sugerido aquí corresponde al utilizado en la encuesta nacional de salud del MINSAL, en donde fue analizado el punto de corte del pCC para la población adulta Chilena

Distinto me parece el valor del indicador ePCR, considerado como un índice de inflamación, sin un claro rol etiológico11. A mi parecer, debe de ser considerado -en ausencia de una inflamación aguda o crónica intercurrente- como un indicador de la gravedad del proceso aterotrombótico, que reconoce a procesos inflamatorios en su secuencia. Si bien la identificación de una CPCR elevada en estudios poblacionales no significa un aporte significativo a la capacidad predictiva cardiovascular ${ }^{12}$, a nivel individual como lo señala Acevedo y cols, puede ser de utilidad para reclasificar el riesgo cardiovascular individual, lo que debería ser demostrado en estudios prospectivos en que los objetivos terminales sean la incidencia de eventos clínicos cardiovasculares.

El estudio Júpiter13, prospectivo, randomizado y controlado, demostró que sujetos sin patología cardiovascular previa y con niveles de colesterol de LDL bajos, pero con elevados niveles de ePCR, se beneficiaron significativamente con el tratamiento con estatinas; ello ha reforzado el valor de este indicador en el contexto de las estrategias de prevención cardiovascular. Sin embargo, hasta el momento no está claro quienes deben ser sometidos a este examen y si está elevado, qué actitud debería ser tomada Tampoco está claro si la determinación a toda la población es costo efectiva. Posiblemente nuevos estudios deberían orientarse a responder estas preguntas orientadas a la practica clínica.

La determinación de la CIMT carotídea, comolo señala Acevedo y col es un marcador de ateroesclerosis subclínica, que se asocia a los factores de riesgo cardiovasculares tradicionales y es un buen predictor de eventos cardiovasculares especialmente encefálicos. Sin embargo, no informa acerca de la presencia y/o intensidad de los factores de riesgo cardiovaculares modificables, lo que constituye la base de las estrategias de prevención cardiovascular, por lo que este indicador debe ser utilizado como un complemento a la determinación de los factores de riesgo tradicionales y al igual que el ePCR puede ser útil para afinar el riesgo cardiovascular, para cambiar los objetivos e intensificar las estrategias.

Quizás la determinación del CIMT carotídeo pudiera ser considerada como indicador preferencial para evaluar la eficacia de nuestras estrategias de prevención a corto plazo, en especial, en prevención primaria en poblaciones jóvenes, lo que debería ser avalado en investigaciones prospectivas antes de recomendar su utilización en la práctica diaria.

\section{Referencias}

1. ACEVEDO A, ARNAÍZ P, CORBALÁN R, GODOY I, MORALES D, CHALHUB M, et al. Modificación del grosor intima-media carotídeo según factores de riesgo clásicos y sindrome metabólico con o sin inflamación. Rev Chil Cardiol 2009; 28: 337-348

2. SERRANO-RIOS M , CARO JF, CARRAZO R, GUTIERREZ JA. The Metabolic Syndrome at the beginning of the XXI century: Genetic and metabolic approach. Elsevier España 2005: 479.

3. FERRANINI E. Metabolic Syndrome: A solution in search of a problem. J Clin Endocrinol \& Metabolism 2007; 92: 396-398

4. DEKKER JM, GIRMAN C, RHODES T, NIJPELS G,
STEHOUWER CD, BOUTER LM, et al. Metabolic Syndrome and 10 year cardiovascular disease risk in the HOORN STUDY. Circulation 2005; 112: 666-73.

5. GRUNDY SM, BREWER HB, CLEEMAN JI, SMITH SC, LENFANT C. Definition of Metabolic Syndrome. NIH-AHA statement. Arterioescler Thromb Vasc Biol 2004; 24: e13-18

6. VALENZUELAA, OLIVOS C, OLEAA R, MAIZ A, ARTEAGA A, RIGOTTI A, et al. Punto optimo de corte de la circunferencia de cintura ( $\mathrm{pCC}$ ) para la población adulta Chilena y su impacto sobre la prevalencia del Síndrome Metabólico. Hallazgos de la primera encuesta nacional de salud en Chile 2003 ( En fase de publicación) 


\section{A. Arteaga}

7. FORD ES. Risks for all-cause mortality, cardiovascular disease, and diabetes associated with the metabolic syndrome: a summary of the evidence. Diabetes Care 2005; 78: 1769-78.

8. LIU J, GRUNDY SM, WANG W, SMITH SC, VEGA G, WU Z, et al. Ten year risk of cardiovascular incidence related to diabetes, pre-diabetes and Metabolic syndrome. Am. Heart J 2007; 153: 552-8

9. GRUNDY SM, CLEEMAN JI, MERZ CN, BREWER HB JR, CLARK LT, HUNNINGHAKE DB, et al. Implications of recent clinical trials for the National Cholesterol Education Program Adult Treatment Panel III Guidelines. J Am Coll Cardiol. 2004; 44: 720-732

10. KNOWLER WC, BARRETT-CONNOR E, FOWLER SE, HAMMAN RF, LACHIN JM, WALKER EA, et al. Reduction in the incidence of type 2 diabetes with lifestyle intervention or metformin. N Engl J Med 2002; 346: 393-403

11. ELLIOT P, CHAMBER JC, ZHANG W. CLARKER, HOPEWELL JC, PEDEN JF, et al. Genetic Loci associated with $\mathrm{C}$-reactive protein levels and risk of coronary heart disease. JAMA 2009; 302: 92-93

12. MELANDER O, NEWTON-CHEH C, ALMGREN P, HEDBLAD B, BERGLUND G, ENGSTRÖM G, et al. Novel and conventional biomarker For prediction of incidence cardiovascular events in the community. JAMA 2009; 302: 49-57

13. RIDKER PM, DANIELSON E, FONSECA FA, GENEST J, GOTTO AM JR, KASTELEIN JJ, et al. Rosuvastatin to prevent vascular events in men and women with elevated C-reactive protein. N Engl J Med. 2008; 359: 2195-2197. 